\title{
A SACRIFICIAL-POLYMER-BASED TRENCH REFILL PROCESS FOR POST- DRIE SURFACE MICROMACHINING
}

\author{
Christophe G. Courcimault* and Mark G. Allen \\ School of Electrical and Computer Engineering, Georgia Institute of Technology, \\ Atlanta, GA 30332-0269
}

\author{
Joseph P. Jayachandran, Paul A. Kohl, and Sue Ann Bidstrup-Allen \\ School of Chemical Engineering, Georgia Institute of Technology, \\ Atlanta, GA 30332-0269
}

\begin{abstract}
We present a trench refill process allowing post-DRIE surface micromachining by formation of planar silicon dioxide membranes encapsulating etched silicon cavities. This process can be used to fabricate diverse structures such as enclosed silicon channels, structures fabricated on top of movable DRIEetched silicon devices, or could even be considered as the first step toward a low-temperature hermetic encapsulation process. Unlike traditional solvent-based sacrificial dissolution processes, the technology is based on the use of heat-degradable polymers as sacrificial materials. Two polymers were studied: a developmental poly(cyclohexene carbonate) and a commercially-available polypropylene carbonate. Formation of air-filled cavities many microns in depth planarized by encapsulating silicon dioxide layers with nonplanarities less than $150 \mathrm{~nm}$ have been realized. The planarization quality as well as the post-processing capabilities have been demonstrated by surface microfabrication of a nickel-electroplated microactuator, exhibiting feature sizes as small as $1 \mathrm{um}$, over a silicon resonator structure defined by a set of multimicron-deep DRIE silicon trenches.
\end{abstract}

\section{INTRODUCTION}

Many microsystems benefit from a combination of attributes from both bulk and surface micromachining. For example, consider the case where a surface micromachined device is to be fabricated on top of a bulk-etched silicon structure. In most cases, when bulk-micromachining is needed to accomplish device fabrication, the deep etching step is performed at the end of the process [1]. The resulting non-planar topography of the substrate surface makes it difficult to consider further surface-micromachining, especially if the surface micromachined structures have exceedingly fine features. Several processes have been introduced in order to address this problem, including LPCVD silicon dioxide deposition, LPCVD polysilicon deposition, and thermal oxide growth, combined with various etch-back methods $[\mathbf{2 , 3 , 4}]$. The trench-refill process presented here is CMOS compatible and not restricted to very narrow DRIE trenches. The technology is based on the processing of a low-temperature degradable polymer as a sacrificial material. The sacrificial polymer is used here as the refilling material that will transform the trench into a gas-filled (or vacuum-filled) cavity capped with $\mathrm{SiO}_{2}$ after decomposition. The "gas-refilled" trench can then be subjected to a wide range

*Travel support has been generously provided by the Transducers Research Foundation and by the DARPA MEMS and DARPA BioFlips programs. of post-processing, ranging from construction of surface micromachined structures to full encapsulation.

\section{HEAT-DEGRADABLE SACRIFICIAL POLYMERS}

Sacrificial layers are key elements in the fabrication of microsystems, as they allow freeing moving structures or define gaps and cavities. Silicon dioxide has been extensively used as a sacrificial material since its deposition and growth are well understood, and its etching can be very selective to silicon. Metals, such as copper, titanium or chromium are also easily accessible and selectively removable. In some cases, because of the large volumes of sacrificial materials required, metals, oxides or nitrides are not appropriate candidate materials; instead, dissolvable polymers such as photoresists are commonly used. However, the development of a suitable fabrication sequence based on these materials can be quite challenging. Polymer layers may reflow, crack, or shrink under postprocessing conditions such as temperature changes or stress introduced by overcoating materials. Even if this issue can be resolved, long dissolution times in solvents are typically required to remove substantial volumes of polymer; and polymeric residues (e.g., for encapsulation applications) can remain a significant problem.

The process presented in this paper focuses on the use of heatdegradable polymers as sacrificial layers for MEMS applications. Such materials typically decompose into volatiles when heated to appropriate temperatures. Several candidate materials have recently emerged which combine microfabrication processability with thermal decomposition properties. The use of heat-degradable functionalized polynorbornenes and polycarbonates has been introduced for the fabrication of air-gaps $[\mathbf{5 , 6 ]}$, with the application of production of low-dielectric-constant materials for high frequency chip interconnection, a key point highlighted by the National Technology Roadmap for Semiconductors [7]. When heated to temperatures varying between $250^{\circ} \mathrm{C}$ to $400^{\circ} \mathrm{C}$, these materials decompose into volatile compounds. The decomposition reaction of suitable polycarbonates as well as the fabrication of air gaps is described in detail by Jayachandran et al. [8]. The typical fabrication process involving these materials mimics that of a photoresist-based sacrificial polymer process. The polymer is spin-cast onto a substrate from solution, soft-baked to remove excess solvent, and then photodefined or dry-etched to form regions of sacrificial polymer. Various over-coating materials, such as low temperature PECVD silicon dioxide, polyimides, or epoxies, are then deposited or cast onto the sacrificial polymer. An interesting characteristic of these overcoats is that they have been demonstrated to be permeable to the decomposition products of the polymer. Therefore, upon heating, the polymer decomposes and the products diffuse through the overcoat, forming encapsulated channels. These channels can be used as lowdielectric-constant supports for high frequency interconnects or for microfluidic applications. 
In this work, two polycarbonate materials were studied for more advanced MEMS applications. The two materials chosen were poly(cyclohexene carbonate), referred to as PCC; and polypropylene carbonate, referred to as PPC. The latter polymer is commercially available from Promerus, Inc. under the name of Unity 2203P. The chemical structures of the two polymers are shown in Figure 1.

Although planarization results will be shown for both polymers, the fabrication sequence will be described for PCC since the processing of the two polymers is substantially similar. The application of interest is a trench-refill process allowing surface-micromachining after a DRIE step, with the ultimate goal of fabricating multilevel stacked resonators. The fabrication sequence presented in Figure 2 shows how the PCC is temporarily used as a refill material prior to being decomposed through a porous PECVD silicon dioxide layer, which has been deposited on top of it. This decomposition process leaves behind an "empty" trench which is capped by the planarized silicon dioxide layer. Since the inorganic capping layer is not susceptible to solvent-induced wrinkling or cracking, thick sacrificial layers without the usual polymer drawbacks are enabled.
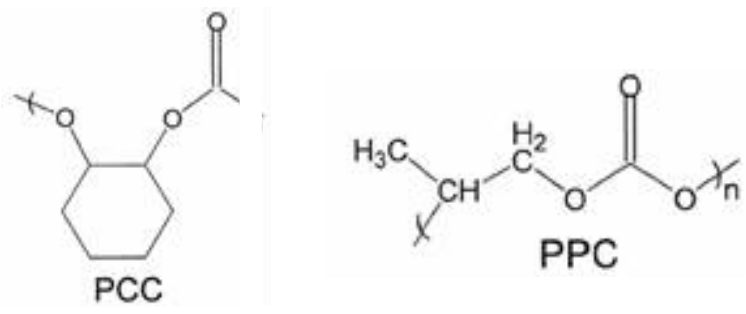

Figure 1. Chemical structures of poly(cyclohexene carbonate), left, and polypropylene carbonate, right.

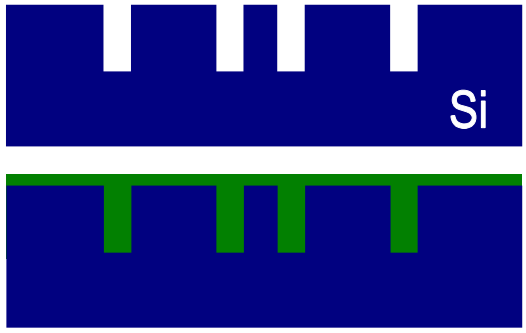

\section{DRE}
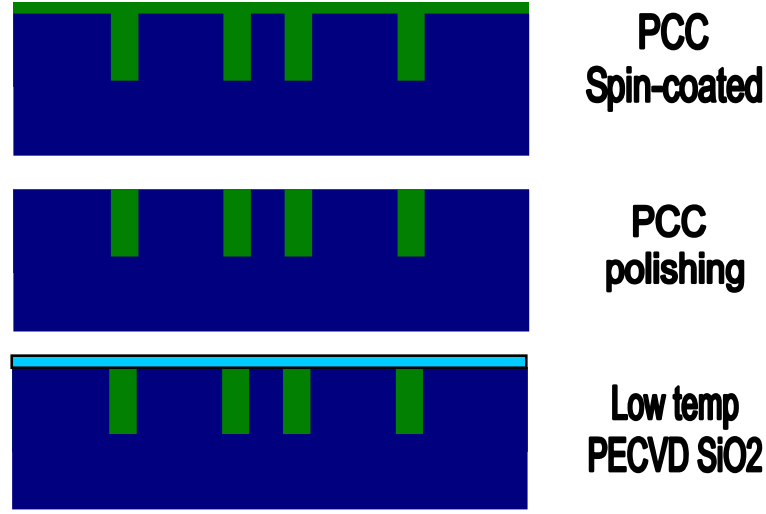

Low temp PECVD SiO2

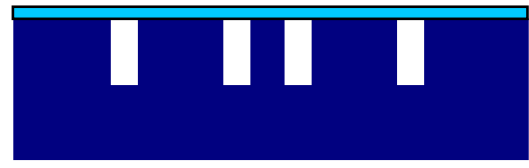

PCC decomposition at $300 \mathrm{C}$

Figure 2. Fabrication Sequence.
The details of the process are given below. Trenches $15 \mu \mathrm{m}$ deep and up to $5 \mu \mathrm{m}$ wide are etched into a silicon wafer using an ICP etch. The sacrificial polymer is then spin-coated on the surface and soft-baked. Due to the excellent planarization properties of the PCC, the material flows into the etched trenches as well as coats the surface of the wafer. The excess polymer in the fields is removed by gentle polishing, leaving the polymer present only in the trenches. A $1.7 \mu \mathrm{m}$ thick layer of porous silicon dioxide is deposited at low temperature $\left(100^{\circ} \mathrm{C}\right)$ using PECVD. The sacrificial polymer is decomposed and vaporized through the porous $\mathrm{SiO}_{2}$. The PCC is decomposed in a nitrogen-purged tube furnace using the following heating steps: the temperature is ramped from room temperature to $150^{\circ} \mathrm{C}$ at $3^{\circ} \mathrm{C} / \mathrm{min}, 150^{\circ} \mathrm{C}$ to $300^{\circ} \mathrm{C}$ at $1^{\circ} \mathrm{C} / \mathrm{min}$ and held at $300^{\circ} \mathrm{C}$ for 3 hours. At this stage of the process, the trenches are free of polymer, and the wafer surface is flat. The silicon dioxide layer can be optionally densified, and the substrate can now be subjected to various micromachining processes such as high temperature steps, photolithography, wet and dry etchings, or material depositions. It should be noted that all of the steps up to this point are lowtemperature and CMOS-compatible.

\section{PLANARIZATION RESULTS}

The planarization of the substrate surface and the range of postprocessing available for surface-micromachining will determine the viability of the trench-refill process. Unlike previous polymer approaches, the process presented here possesses the desirable feature that the trenches are refilled with air. As long as the $\mathrm{SiO}_{2}$ membrane is not removed, a wide variety of post-processing options are available. Figure 3a) shows a cross-sectional scanning electron micrograph of a trench refilled by PCC, polished, and coated with low temperature PECVD oxide. As shown in Figure 3b), the surface of the substrate after oxide deposition but before PCC removal analyzed using a Tencor Alpha-Step surface profilometer presents a nonplanarity of only $115 \mathrm{~nm}$ over the $3 \mu \mathrm{m}$ width of the $15 \mu \mathrm{m}$ deep trench. Depending on the measurement sample, both depressions as well as peaks were observed; but in all cases the magnitude of the nonplanarities was less than $300 \mathrm{~nm}$. The PCC was then thermally decomposed. A cross-sectional view of the trench after decomposition of the PCC through the porous $\mathrm{SiO}_{2}$ is shown in Figure 4a). The trench is free of polymer and residue and the $\mathrm{SiO}_{2}$ membrane has neither visibly sagged nor broken. Furthermore, the profilometer trace shown in Figure 4b) confirms that the $\mathrm{SiO}_{2}$ membrane is not affected by the decomposition step and reaffirms the quality of the surface planarization. Again, depending on the measurement sample, both depressions as well as peaks were observed, but in all cases the magnitude of the nonplanarities was less than $300 \mathrm{~nm}$. In this particular case, the surface of the substrate exhibits a $120 \mathrm{~nm}$ depression. A similar planarization trace for PPC is shown in Figure 5. Here, after decomposition, an additional postprocessing metal layer of $\mathrm{Ti} / \mathrm{Cu} / \mathrm{Ti}$, with the $\mathrm{Cu}$ layer $4 \mu \mathrm{m}$ in thickness, has been deposited, illustrating the robustness of the capping $\mathrm{SiO}_{2}$ layer. The planarization of the resultant surface exhibits a $300 \mathrm{~nm}$ nonplanarity over the width of the trench.

\section{POST-PROCESSING DEMONSTRATION}

In order to demonstrate the capabilities of post-DRIE surfacemicromachining using this approach, a nickel electroplated microactuator is fabricated on top of the "air-refilled" cavities. The fabrication sequence is presented in Figure 6. The $\mathrm{SiO}_{2}$ layer, used for encapsulation of the silicon cavities, also acts as a sacrificial layer for the microactuator. A seed layer (Ti: $15 \mathrm{~nm} / \mathrm{Cu}: 100 \mathrm{~nm} / \mathrm{Ti}: 15 \mathrm{~nm})$ is then deposited on top of the oxide and a 10 micron thick Shipley 

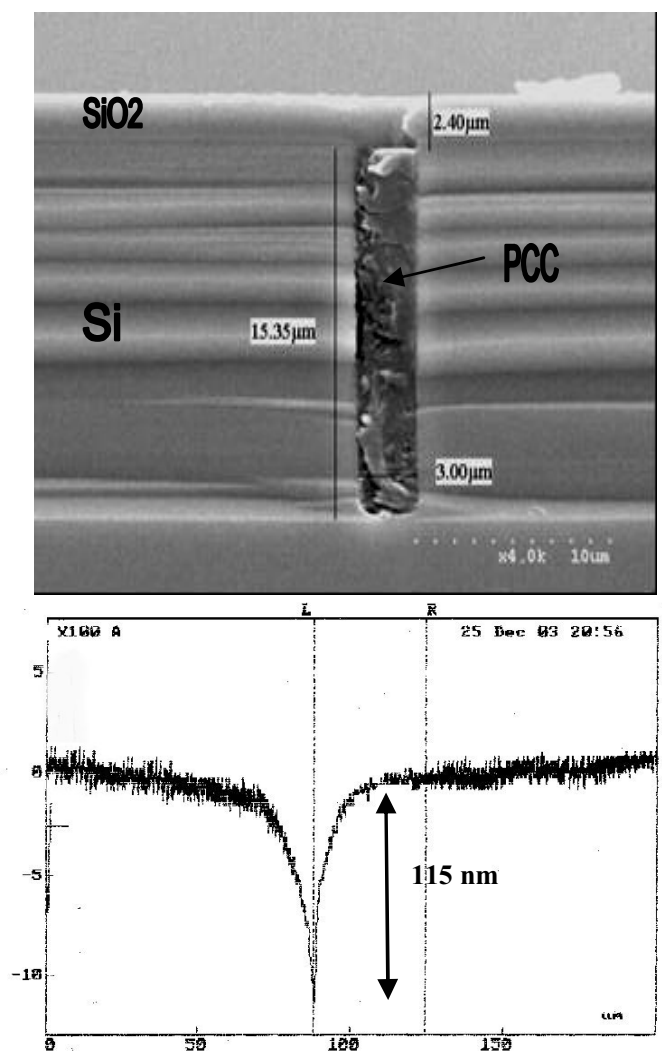

Figure 3. a) SEM or the trench after PCC refill, polishing and low-temperature $\mathrm{SiO}_{2}$ deposition. b) Profile of the substrate surface.
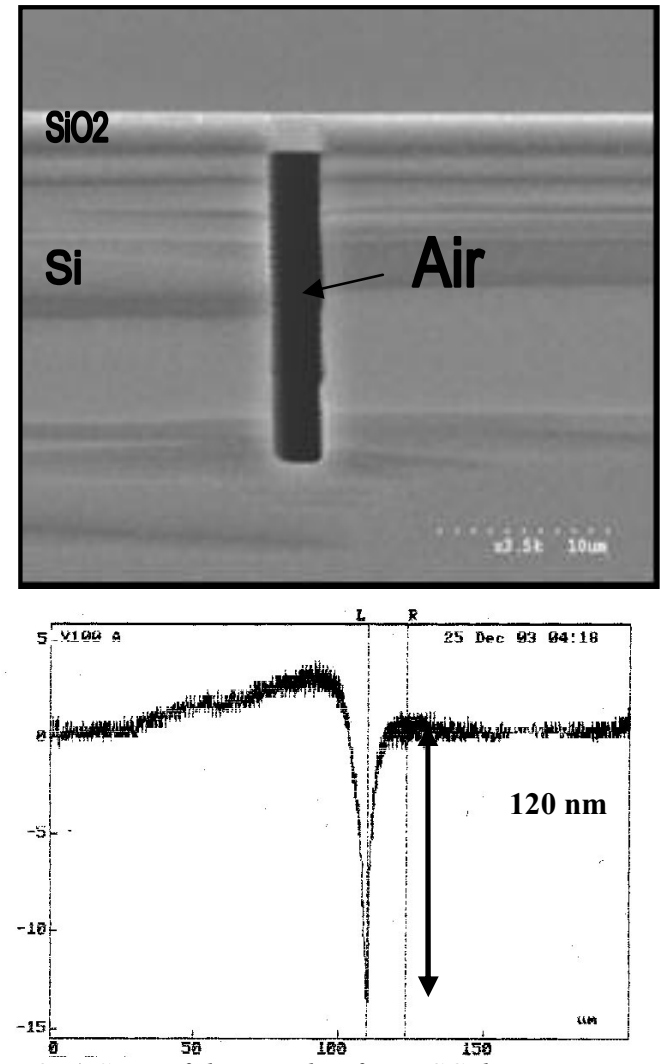

Figure 4. a) SEM of the trench, after PCC decomposition. b) Profile of the substrate surface above "empty" trench. photoresist (SPR220-7) is spun, soft-baked, exposed, post-baked and developed to define an electroplating mold. Using a sulfamate nickel bath, a nine micron thick nickel layer is electroplated. After removal of the mold, the silicon dioxide layer is time-etched using a buffered oxide etch solution $(6: 1)$ to release the microactuator and re-open the silicon trenches.

The microactuator is electrostatically actuated by means of a set of comb-drive electrodes. Figure 7 shows an SEM of the interdigitated fingers. Due to the excellent substrate planarization, high resolution photolithography can be performed, allowing fine feature definition. Note the gap between fingers is in the order of 1.1 microns, over a thickness of nine microns (aspect-ratio: 1:8). Figure $8 \mathrm{a}$ shows an overall view of the device, and Figure $8 \mathrm{~b}$ displays the microactuator shuttle free-standing above an empty and clear trench. The microactuator has also been electrically tested. In this device, the 1.7 micron thick silicon dioxide layer serves two purposes: first, for encapsulating the trenches during the microactuator fabrication; and second, to provide electrical insulation of the actuator electrodes. The microactuator exhibits lateral displacement between the interdigitated finger gap. The full range of displacement, 1.1 micron, is obtained under the application of $30 \mathrm{~V}$ DC voltage. The insulating properties of the porous, low temperature PECVD oxide have also been examined. Pad-to-pad bias voltages of up to $100 \mathrm{~V}$ have been applied to the device without breaking down the 1.7 micron layer of $\mathrm{SiO}_{2}$. this demonstration device verifies the suitability of the trench-refill process for subsequent surface micromachining.

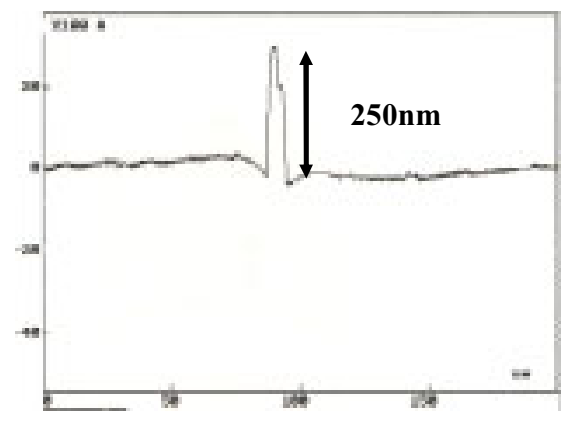

Figure 5. Profile of the substrate surface above "empty" trench after PPC decomposition and deposition of a 4 micron $\mathrm{Ti} / \mathrm{Cu} / \mathrm{Ti}$ layer.

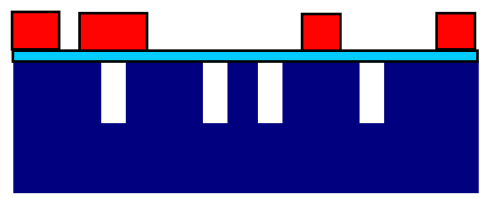

\section{PRmold}
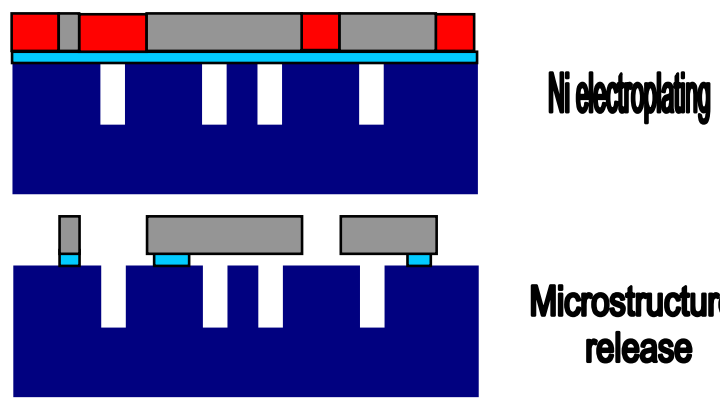

Microstructure

release

Figure 6. Fabrication sequence of the electroplated actuator above the DRIE trenches. 


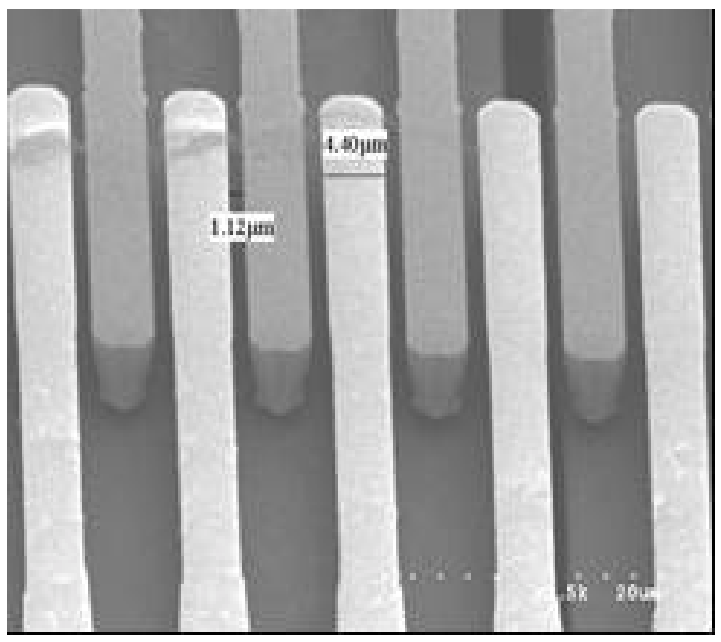

Figure 7. Electroplated nickel though photoresist mold. The fingers are 4.4 um wide, 9 um thick, and spaced by a 1.1 um gap $(A . R=1: 8)$.

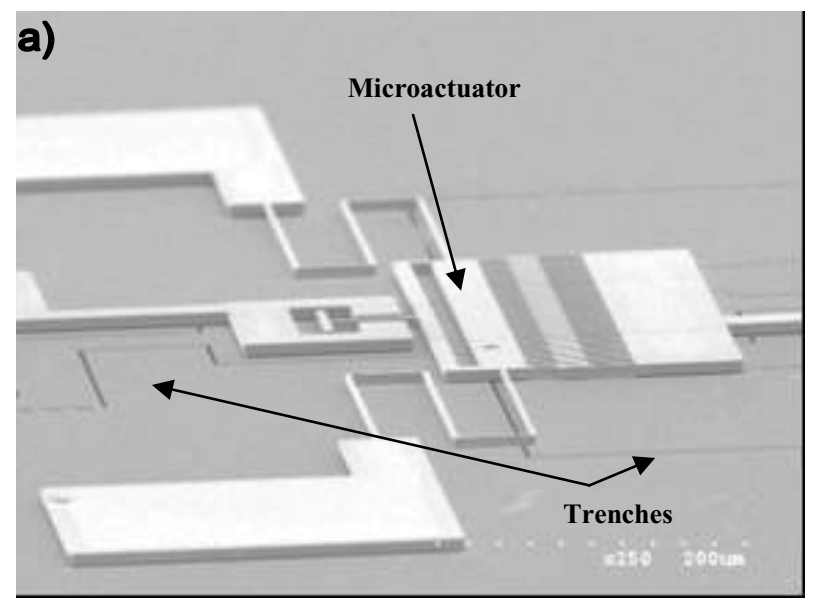

b) Free-standing shuttle

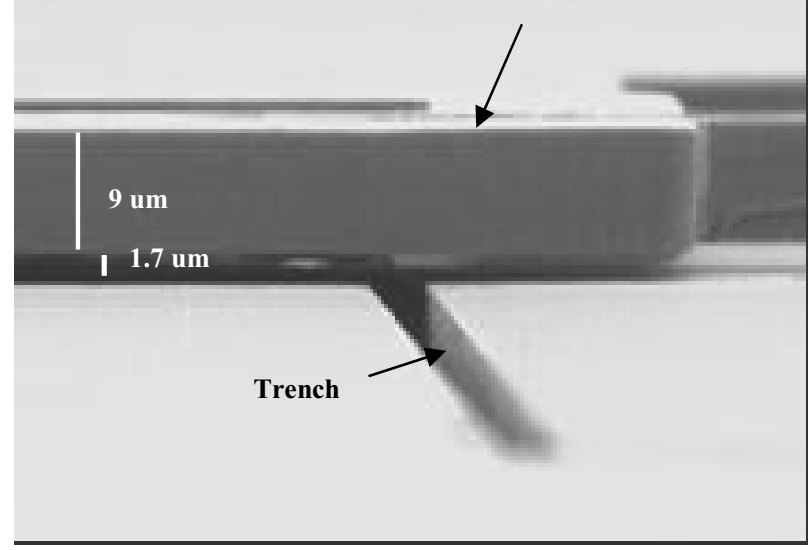

Figure 8 a) Overall view of the microactuator fabricated over the trenches. b) Released microactuator shuttle standing on top of a trench.

\section{CONCLUSIONS}

The formation of air cavities from DRIE silicon trenches encapsulated by PECVD silicon dioxide after thermal decomposition of various polycarbonates was demonstrated as a suitable method for post-DRIE surface micromachining. The process is CMOScompatible due to the low temperature heat degradable polycarbonate, is applicable to wider trenches than previouslyreported refill processes, and does not affect the size of the trenches. Excellent planarization of the process was observed, allowing the fabrication of micron-scale features directly on top of the sealed trenches. Although the application presented here was for post-DRIE surface micromachining, further applications such as large surface planarization, silicon buried air channels and vacuum encapsulation of microsystems are enabled by this process.

\section{ACKNOWLEDGMENTS}

This work was supported in part by the U.S. Defense Advanced Research Projects Agency under the NMASP Program. Microfabrication was carried out in the Georgia Tech Microelectronics Research Center with the assistance of the staff.

\section{REFERENCES}

1. T. Muller et al., "An industrial CMOS process family adapted for the fabrication of smart silicon sensors", Sensors and Actuators 84 (2000), pp. 126-133

2. C. Zhang, K. Najafi, "Fabrication of thick silicon dioxide layers using DRIE, oxidation and trench refill", Micro Electro Mechanical Systems, 2002. The Fifteenth IEEE International Conference on, 2024 Jan. 2002, pp $160-163$.

3. K. Shenai., "A novel planarization technique using polysilicon refill, polysilicon oxidation and oxide etchback" IEEE transactions on electron devices, vol.40, no.2, February 1993, pp 459-463.

4. F. Ayazi, K. Najafi, "High aspect-ratio combined poly and singlecrystal silicon (HARPSS) MEMS technology", Journal of Microelectromechanical Systems, vol. 9 , Sept. 2000 pp 288-294.

5. Paul A. Kohl, et al., "Air-gaps for electrical interconnections", Electrochemical and Solid-State Letters, 1 (1) 49-51 (1998).

6. Hollie A. Reed, et al., "Fabrication of microchannels using polycarbonates as sacrificial materials", J. Micromech. and Microeng., 11 (2001) 733-737.

7. The National Technology Roadmap for Semiconductors, Semiconductor Industry Association, San Jose, CA, 1997.

8. J. Jayachandran, et al., "Air-channel fabrication for microelectromechanical systems via sacrificial photosensitive polycarbonates", Journal of Microelectromechanical Systems, vol. 12, no. 2, April 2003, pp 147-159. 